\title{
Imaging in Inflammatory Bowel Diseases - Magnetic Resonance Imaging
}

\author{
Hans Herfarth $^{a}$ Andreas G. Schreyer ${ }^{b}$ \\ ${ }^{a}$ Division of Gastroenterology and Hepatology, University of North Carolina, Chapel Hill, N.C., USA; ${ }^{b}$ Department of \\ Radiology, University Regensburg, Regensburg, Germany
}

\section{Key Words}

Magnetic resonance enterography · Magnetic resonance imaging · Inflammatory bowel diseases · Crohn's disease

\begin{abstract}
Diagnostic imaging techniques play an important role in the diagnosis and management of patients with inflammatory bowel diseases (IBDs). The approach should be guided by considerations of diagnostic accuracy, concerns about patient exposure to ionizing radiation, local expertise and tolerance of the endoscopic and/or imaging technique. In regard to the clinical diagnostic value (sensitivity, specificity and accuracy), no significant differences exist between CT and MRI for the evaluation of the extent of inflammation, stricturing, penetrating disease or extraluminal complications such as abscesses. Due to the absence of radiation exposure, MRI of the intestine is recommended as the first-line imaging modality in patients with suspected or established IBD. The focus of this review is the latest developments in MRI techniques to detect IBDs. Specifically, the use of new indices for the grading of inflammation or assessing bowel damage as well as innovative experimental approaches such as diffusion-weighted imaging or magnetization-transfer $M R I$ to evaluate and quantify the degree of intestinal inflammation and fibrosis in stricturing Crohn's disease are discussed.

(c) 2015 S. Karger AG, Basel
\end{abstract}

\section{Introduction}

Currently, European guidelines recommend the preferential use of MRI compared to CT for evaluation of the small bowel in patients with inflammatory bowel diseases (IBDs) [1]. Mainly, this choice of MRI over CT hinges upon the lack of exposure to radiation since, overall, these cross-sectional imaging modalities are comparable both in sensitivity and specificity in diagnosing inflammatory Crohn's disease (CD) activity, fistula, strictures and abscesses [2-4]. A recent meta-analysis of 6 trials comparing MRI and CT revealed the pooled sensitivity and specificity for MR enterography (MRE) in detecting active CD to be $87.9 \%$ (95\% confidence interval (CI) 81.8-92.5) and 81.2\% (95\% CI 71.988.4), respectively, compared to the pooled sensitivity and specificity of CT enterography (CTE) of $85.8 \%$ (95\% CI 79.2-90.9) and $83.6 \%$ (95\% CI 75.3-90.1), respectively [5]. All MREs were performed using a 1.5-T imager and 4 of the 6 CTEs were performed using a 64-detector row CT scanner except in 2 studies, which used a 16-row detector CT. The meta-analysis also revealed no difference in either sensitivity and specificity in detecting fistula, strictures or abscesses. However, a significant heterogeneity of the studies was detected, which is almost certainly due to the fact that the studies used different criteria to define the severity and extent of inflammation

\section{KARGER 125}

( 2015 S. Karger AG, Base

$0257-2753 / 15 / 0335-0026 \$ 39.50 / 0$ 
Table 1. Published prospective and retrospective studies comparing CT and MRI in the evaluation of patients with IBD MRI signs of bowel inflammation and/or damage [7]

\begin{tabular}{ll}
\hline General radiological signs (also applied in CT) & Specific MRI signs \\
\hline Abscess & ADC \\
$\begin{array}{l}\text { Comb sign (prominent mesenteric vasculature in } \\
\text { an inflamed segment) }\end{array}$ & $\begin{array}{l}\text { DWI hyperintensity } \\
\text { Enhancement kinetics after gadolinium } \\
\text { administration (e.g. wall enhancement, fat or lymph } \\
\text { Fat edema }\end{array}$ \\
$\begin{array}{l}\text { Fibro-fatty proliferation } \\
\text { Free fluid }\end{array}$ & Motility (using MRI cinematography) \\
$\begin{array}{l}\text { Lymph node size and number } \\
\text { Mucosal lesions }\end{array}$ & \\
$\begin{array}{l}\text { Stricture (decrease in bowel lumen with or } \\
\text { without prestenotic dilation) }\end{array}$ & \\
Wall thickness & \\
\hline
\end{tabular}

since there are no single or even widely accepted radiologic scoring indexes of inflammation for cross-sectional imaging results. The absence of a reproducible radiological IBD index also signifies that the gastroenterologist strongly depends on the radiologist's expertise in reading and interpreting pathological enterography results. Thus, gastroenterologists treating IBD patients should use the imaging techniques that are supported by the best local radiological expertise rather than simply follow the recommended guidelines.

Aside from the advantage of being radiation-free, MRI also has a disadvantage compared to CT. MRI technology still requires significantly longer acquisition times. Therefore, in acute situations (e.g. acute abdomen) or in older patients or patients with difficulties holding their breath, CT is still the preferred imaging method [1]. A recent study revealed that in CD patients $(\mathrm{n}=53)$ with an acute onset of severe abdominal pain, the evaluation with a conventional abdominal 16-slice multidetector CT revealed findings similar to those obtained with a dedicated MRE [6]. Overall, the diagnosis of inflammation based on typical radiological signs of bowel wall thickening, stenosis of the lumen, increased contrast media uptake, enlarged local lymph nodes $(>5$ $\mathrm{mm}$ in the short axis) and local mesenteric injection was not significantly different between CT (69.4\%) and MRE (71.4\%). Colonic inflammation was diagnosed in $30.2 \%$ of patients based on CT and in $29.0 \%$ of them based on MRE. The difference in the detection of lymph nodes was significant (CT: $\mathrm{n}=49$ and MRE: $\mathrm{n}=27$ ), whereas the differences between fistula (CT: $\mathrm{n}=25$ and MRE: $\mathrm{n}=27$ ) or abscesses (CT and MRE: $\mathrm{n}=32$ ) detection were not significant.

Imaging in Inflammatory Bowel Diseases

\section{MRI Scoring Systems of Inflammation}

Classic radiologic signs of inflammation commonly used in MRI and CT cross-sectional imaging are bowel wall thickening, contrast wall enhancement and visible ulcerations (table 1). However, a recent meta-analysis on MRE indications for the detection of inflammation and intestinal damage in $\mathrm{CD}$ has revealed a total of 22 signs to characterize inflammation [7]. This illustrates the difficulty to agree on a uniform index for evaluation of inflammation with MRI. Fourteen MRI-based disease activity indices have been described and proposed [8]. So far, only the Magnetic Resonance Index of Activity (MaRIA) has been externally evaluated and seems to have gained traction as an index for clinical studies [9-12]. The MaRIA score is a composite score of pre- and post-contrast wall signal intensity for wall contrast enhancement, the thickening of the bowel and the presence of ulcers and edema. The score for a specific bowel segment (ileum, ascending colon, transverse colon, descending colon, sigmoid and rectum) is calculated using the following, rather complex, formula:

$$
\begin{aligned}
\text { MaRIA }(\text { of a segment })= & (1.5 \times \text { wallthickening }(\mathrm{mm}))+ \\
& (0.02 \times \text { relative contrast en- } \\
& \text { hancement })+(5 \times \text { edema })+ \\
& (10 \times \text { ulceration }) .
\end{aligned}
$$

The global MaRIA score is then computed as the sum of the segmental MaRIA scores. The MaRIA score correlates with an endoscopic score of intestinal inflammation (CD Endoscopic Index of Severity (CDEIS)) and is also moderately correlated with the Harvey-Bradshaw index 
and C-reactive protein as a serologic marker of disease activity [9]. More recently, this score was used to measure response to therapy and mucosal healing in patients with CD [12]. In this 4-center Spanish study, 48 patients with active CD (CD Activity Index $>150$ ) confirmed by ileocolonoscopy (CDEIS $\geq 7$ and presence of ulcers in at least one segment) were treated with either corticosteroids $(\mathrm{n}=14,29.2 \%)$ or agents that acted as antibodies to tumor necrosis factor ( $\mathrm{n}=34,70.8 \%$ ), for 12 weeks. MRI and ileocolonoscopy were performed before and after the treatment. The prediction of ulcer healing on a per segment basis with a segmental MaRIA $<11$ had a sensitivity, specificity, positive predictive value, negative predictive value and accuracy of $94,69,94,67$ and $90 \%$, respectively. Mucosal healing could be predicted similarly with a segmental MaRIA score of $<7$ with a sensitivity, specificity, positive predictive value, negative predictive value and accuracy of $85,78,92,63$ and $83 \%$, respectively. The findings suggest that this index might facilitate a non-invasive evaluation of mucosal healing in clinical trials. Additionally, in case mucosal healing becomes an established therapeutic goal in clinical practice, a recurrent evaluation with MRI to evaluate both the small and large intestines for ongoing inflammation seems to be more feasible and safer than recurrent ileocolonoscopies. However, from the practical standpoint, it is important to note that for a complete assessment of both the small and large bowels, the patient not only needs to be prepped with oral contrast but a rectal enema also needs to be applied for colonic distension (800-1,500 ml volume). Without colonic distension by the application of an enema, the diagnostic accuracy in evaluating the colonic mucosa for inflammatory lesions and strictures significantly decreases. As has recently been shown by Friedrich et al. [13], the sensitivity in detecting colonic inflammation decreases from $79 \%$ with a specificity of $96 \%$ with an water enema application to a sensitivity of $38 \%$ and specificity $99 \%$, respectively, without the water application. However, whereas rectal enemas in the setting of MRI and CT examinations are often applied in Europe, these are not very welcomed in radiologic centers in the USA due to the need for enema application on the examination table, which results in longer examination times and the risk of incontinence, which then necessitates time-consuming room cleaning (Loftus and Herfarth, personal communication).

More recently, a scoring system to evaluate disease progression by assessing structural bowel damage, including stricturing lesions, penetrating lesions and surgical resection, (the Lemann Index) has been evaluated in a
Table 2. Potential advantages of added DWI compared to only 'conventional' MRI

No need for oral or intravenous contrast

Quantification of inflammation (potential biomarker)

Characterization of inflammation versus fibrosis (using ADC)

multicenter study [14]. This system is based mainly on MRI/MRE imaging results and may in the future facilitate the evaluation of the efficacy of therapies in reversing or halting bowel damage. However, the score has yet to be validated independently, and the reproducibility of the MRI scoring system has to be confirmed across multiple sites [15]. Given the high costs of MRI (at least in the USA, MRI is significantly more expensive than CT), it would also be desirable if the Lemann score could be reproduced using low-dose radiation CT imaging.

\section{New Developments in IBD MRI Diagnostics: Diffusion-Weighted MRI}

A specific MRI technology is the diffusion-weighted imaging (DWI). The quantitative measurement for this technology is termed 'apparent diffusion coefficient' (ADC). The DWI technology, which was initially used in neuroimaging, depicts differences in the motion of water molecules in body tissues [16]. The ADC value and thus the mobility of water molecules decrease with increased tissue cellularity such as inflammation or malignancy. The ADC value may also be used to grade tissue differentiation as shown recently in patients with rectal cancer [17]. The ADC significantly decreased with poorer differentiation of the cancer.

Several studies have evaluated the application of DWMRI in IBD [11, 16, 18-25]. Overall it seems that the clinical value of DWI in addition to a conventional MRI or MRE still requires further elucidation, but the studies published thus far point toward several additional advantages of incorporating DWI to the standard MRI (table 2). Kim et al. [16] studied 55 patients with suspected CD prospectively using conventional MRI and DWI and compared the findings to ileocolonoscopy results. Of these, 44 patients were finally diagnosed with CD. Overall, the sensitivity for detecting mild inflammation in this study was increased with DWI compared to conventional MRI; however, the specificity was lower, especially, in the colorectum (table 3 ). The authors discuss that the low specificity might be due to the fact that the study was performed
28

Dig Dis 2015;33(suppl 1):26-31

DOI: $10.1159 / 000437036$
Herfarth/Schreyer 
Table 3. Sensitivity and specificity in detection of inflammation by DWI MRE compared to conventional MRE (ND; not done) [16]

\begin{tabular}{|c|c|c|c|c|c|c|}
\hline & \multicolumn{3}{|c|}{ Sensitivity } & \multicolumn{3}{|c|}{ Specificity } \\
\hline & MRI, \% & MRI + DWI, \% & $\mathrm{p}$ value & MRI, \% & MRI + DWI, \% & $\mathrm{p}$ value \\
\hline Inflammation & 62 & 83 & $<0.001$ & 94 & 60 & $<0.001$ \\
\hline Deep ulcers & 97 & 97 & & ND & ND & \\
\hline $\begin{array}{l}\text { Aphthae, erythema, } \\
\text { edema } \\
\text { Location inflammation }\end{array}$ & 18 & 62 & $<0.001$ & ND & ND & \\
\hline Terminal ileum & 91 & 94 & & & & \\
\hline Colorectum & 45 & 76 & $<0.001$ & 96 & 57 & $<0.001$ \\
\hline
\end{tabular}

Fig. 1. Proposed MRI predictors for the degree of inflammation and fibrosis in resected Crohn's intestinal segments.

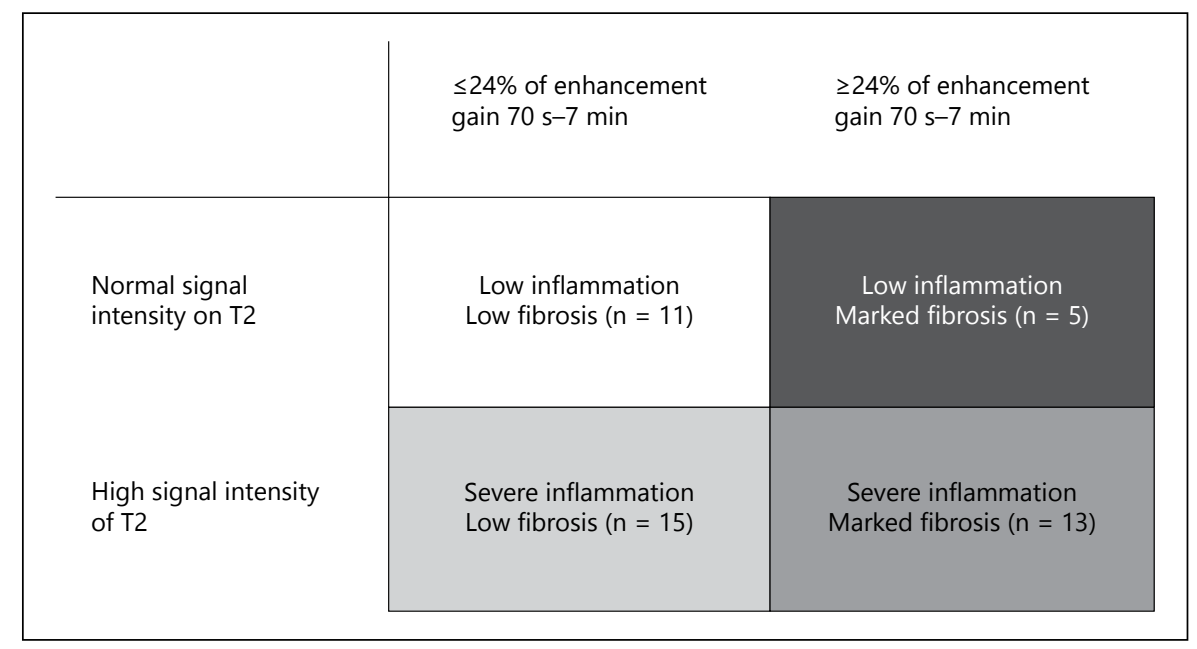

without rectal contrast similar to a previous DWI study in CD patients, which also reported increased sensitivity in the detection of inflammation using DWI but a specificity of $62 \%$ [23]. A study similar to the one performed by Friedrich et al. [13] comparing conventional MRI with and without rectal enema seems to be warranted for further evaluation of the clinical application of DWI. Pilot studies have also shown that DWI and ADC may be used to quantify intestinal inflammation and characterize the degree of fibrosis in stenotic disease [11, 19, 21, 25].

\section{Evaluation of Strictures in CD}

Around $30-50 \%$ of all CD patients develop stricturing bowel disease during the course of their illness. These strictures are characterized initially by an inflammatory component; however, in the setting of prolonged inflammation, excessive production of extracellular matrix components occurs and strictures become fibrotic over time [26]. CT and MRI have sensitivities in the range of $90 \%$ to detect strictures with and without prestenotic dilation, but so far no imaging modality can reliably evaluate the extent of fibrosis. Also, the addition of positron emission tomography to CT or MRI has so far not achieved a clinically usable differentiation of inflammation versus fibrosis in strictures [27]. However, the technology of MRI is further evolving and might offer the diagnostic accuracy for the delineation between inflammation and fibrosis in the bowel wall in the near future.

Rimola et al. [28] recently evaluated the value of different MRI variables to predict fibrosis and inflammation in comparison with histology in a retrospective analysis of 41 patients undergoing elective surgery for bowel resection. They found that inflammation correlated significantly with the following 4 parameters: hypersignal on T2, mucosal enhancement, ulcerations and blurred margins. The degree of fibrosis correlated with 3 MRI param- 
eters: percentage of enhancement gain, the pattern of enhancement at $7 \mathrm{~min}$ and the presence of stenosis. They proposed a model that, based on these parameters, was able to predict the degree of inflammation and fibrosis in a strictured segment (fig. 1). These parameters should now be evaluated in a multicenter prospective study with a unified imaging protocol. In such a prospective study, the comparability of the imaging results of MRI scanners from different manufacturers can also addressed, since the results may be quite different as shown in the past in other specialties such as oncology [29].

Other technologies to evaluate strictures are DWI (see above) and 'magnetization transfer' (MT)-MRI [30]. Whereas DWI centers on the mobility of water molecules in the context of the surrounding tissue, MT-MRI focuses on the interactions between the protons of free water and those of large immobilized macromolecules, such as collagen or immobilized phospholipid cell membranes in tissue [31]. Animal experiments using the purified peptidoglycan-polysaccharide rat colitis model have demonstrated the feasibility of MT-MRI in measuring the degree of intestinal fibrosis [32-34]. Initial data in patients confirm the potential of this imaging modality in revealing significant differences of the MT ratio in gut segments with fibrosis compared to normal or inflamed tissue [35, 36].

\section{Summary}

In the last decade, MRI has been established as the preferred imaging technology in patients with IBD. Generally recognized scores for assessing the degree and extent of inflammation, such as the MaRIA score or the Lemann score, have been proposed and should now be broadly validated to assure reliability and reproducibility. Technologies, such as DWI and MT-MRI, are promising endeavors that add information to conventional MRI scans in IBD. Further validation of these technologies is necessary, but these technologies may be able to quantify the degree of inflammation and fibrosis in the near future.

\section{Disclosure Statement}

The authors have no conflicts of interest to declare.

\section{References}

1 Panes J, Bouhnik Y, Reinisch W, et al: Imaging techniques for assessment of inflammatory bowel disease: joint ECCO and ESGAR evidence-based consensus guidelines. J Crohns Colitis 2013;7:556-585.

2 Herfarth $\mathrm{H}$ : Update imaging in inflammatory bowel diseases. Dig Dis 2013;31:345-350.

3 Panés J, Bouzas R, Chaparro M, et al: Systematic review: the use of ultrasonography, computed tomography and magnetic resonance imaging for the diagnosis, assessment of activity and abdominal complications of Crohn's disease. Aliment Pharmacol Ther 2011;34: 125-145.

4 Horsthuis K, Bipat S, Bennink RJ, et al: Inflammatory bowel disease diagnosed with US, MR, scintigraphy, and CT: meta-analysis of prospective studies. Radiology 2008;247:64-79.

5 Qiu Y, Mao R, Chen BL, et al: Systematic review with meta-analysis: magnetic resonance enterography vs. computed tomography enterography for evaluating disease activity in small bowel Crohn's disease. Aliment Pharmacol Ther 2014;40:134-146.

6 Schreyer AG, Hoffstetter P, Daneschnejad M, et al: Comparison of conventional abdominal CT with MR-enterography in patients with active Crohn's disease and acute abdominal pain. Acad Radiol 2010;17:352-357.

7 Church PC, Turner D, Feldman BM, et al: Systematic review with meta-analysis: mag- netic resonance enterography signs for the detection of inflammation and intestinal damage in Crohn's disease. Aliment Pharmacol Ther 2015;41:153-166.

8 Rimola J, Ordás I, Rodríguez S, et al: Imaging indexes of activity and severity for Crohn's disease: current status and future trends. Abdom Imaging 2012;37:958-966.

9 Rimola J, Ordás I, Rodriguez S, et al: Magnetic resonance imaging for evaluation of Crohn's disease: validation of parameters of severity and quantitative index of activity. Inflamm Bowel Dis 2011;17:17591768.

10 Rimola J, Rodriguez S, García-Bosch O, et al: Magnetic resonance for assessment of disease activity and severity in ileocolonic Crohn's disease. Gut 2009;58:1113-1120.

11 Hordonneau C, Buisson A, Scanzi J, et al: Diffusion-weighted magnetic resonance imaging in ileocolonic Crohn's disease: validation of quantitative index of activity. Am J Gastroenterol 2014;109:89-98.

12 Ordás I, Rimola J, Rodríguez S, et al: Accuracy of magnetic resonance enterography in assessing response to therapy and mucosal healing in patients with Crohn's disease. Gastroenterology 2014;146:374-382.e1.

13 Friedrich C, Fajfar A, Pawlik M, et al: Magnetic resonance enterography with and without biphasic contrast agent enema compared to conventional ileocolonoscopy in patients with Crohn's disease. Inflamm Bowel Dis 2012;18:1842-1848.

14 Pariente B, Mary JY, Danese S, et al: Development of the Lémann index to assess digestive tract damage in patients with Crohn's disease. Gastroenterology 2015;148:52-63.e3.

15 Ananthakrishnan AN, Hanauer SB: The Holy Grail, or only half way there? Gastroenterology 2015;148:8-10.

16 Kim KJ, Lee Y, Park SH, et al: Diffusionweighted MR enterography for evaluating Crohn's disease: how does it add diagnostically to conventional MR enterography? Inflamm Bowel Dis 2015;21:101-109.

17 Akashi M, Nakahusa Y, Yakabe T, et al: Assessment of aggressiveness of rectal cancer using 3-T MRI: correlation between the apparent diffusion coefficient as a potential imaging biomarker and histologic prognostic factors. Acta Radiol 2014;55:524-531.

18 Buisson A, Joubert A, Montoriol PF, et al: Diffusion-weighted magnetic resonance imaging for detecting and assessing ileal inflammation in Crohn's disease. Aliment Pharmacol Ther 2013;37:537-545.

19 Kiryu S, Dodanuki K, Takao H, et al: Freebreathing diffusion-weighted imaging for the assessment of inflammatory activity in Crohn's disease. J Magn Reson Imaging 2009; 29:880-886. 
20 Neubauer H, Pabst T, Dick A, et al: Smallbowel MRI in children and young adults with Crohn disease: retrospective head-to-head comparison of contrast-enhanced and diffusion-weighted MRI. Pediatr Radiol 2013;43: 103-114.

21 Oto A, Kayhan A, Williams JT, et al: Active Crohn's disease in the small bowel: evaluation by diffusion weighted imaging and quantitative dynamic contrast enhanced MR imaging. J Magn Reson Imaging 2011;33: 615-624.

22 Oto A, Zhu F, Kulkarni K, et al: Evaluation of diffusion-weighted MR imaging for detection of bowel inflammation in patients with Crohn's disease. Acad Radiol 2009;16:597-603.

23 Oussalah A, Laurent V, Bruot O, et al: Diffusion-weighted magnetic resonance without bowel preparation for detecting colonic inflammation in inflammatory bowel disease. Gut 2010;59:1056-1065.

24 Ream JM, Dillman JR, Adler J, et al: MRI diffusion-weighted imaging (DWI) in pediatric small bowel Crohn disease: correlation with MRI findings of active bowel wall inflammation. Pediatr Radiol 2013;43:1077-1085.
25 Tielbeek JA, Ziech ML, Li Z, et al: Evaluation of conventional, dynamic contrast enhanced and diffusion weighted MRI for quantitative Crohn's disease assessment with histopathology of surgical specimens. Eur Radiol 2014; 24:619-629.

26 Rieder F, Zimmermann EM, Remzi FH, et al: Crohn's disease complicated by strictures: a systematic review. Gut 2013;62:1072-1084.

27 Perlman SB, Hall BS, Reichelderfer M: PET/ CT imaging of inflammatory bowel disease. Semin Nucl Med 2013;43:420-426.

28 Rimola J, Planell N, Rodríguez S, et al: Characterization of inflammation and fibrosis in Crohn's disease lesions by magnetic resonance imaging. Am J Gastroenterol 2015;110: 432-440.

29 Ihalainen TM, Lönnroth NT, Peltonen JI, et al: MRI quality assurance using the ACR phantom in a multi-unit imaging center. Acta Oncol 2011;50:966-972.

30 Al-Hawary M, Zimmermann EM: A new look at Crohn's disease: novel imaging techniques. Curr Opin Gastroenterol 2012;28:334-340.

31 Shah B, Anderson SW, Scalera J, et al: Quantitative MR imaging: physical principles and sequence design in abdominal imaging. Radiographics 2011;31:867-880.

32 Adler J, Rahal K, Swanson SD, et al: Anti-tumor necrosis factor a prevents bowel fibrosis assessed by messenger RNA, histology, and magnetization transfer MRI in rats with Crohn's disease. Inflamm Bowel Dis 2013;19:683-690.

33 Adler J, Swanson SD, Schmiedlin-Ren P, et al Magnetization transfer helps detect intestinal fibrosis in an animal model of Crohn disease. Radiology 2011;259:127-135.

34 Dillman JR, Swanson SD, Johnson LA, et al: Comparison of noncontrast MRI magnetization transfer and T2-weighted signal intensity ratios for detection of bowel wall fibrosis in a Crohn's disease animal model. J Magn Reson Imaging 2014, Epub ahead of print.

35 Pazahr S, Blume I, Frei P, et al: Magnetization transfer for the assessment of bowel fibrosis in patients with Crohn's disease: initial experience. MAGMA 2013;26:291-301.

36 Helvie K, Dhanani M, Al-Hawary MM, et al: Magnetization-transfer MRI as a non-invasive method of assessing fibrotic intestinal strictures in patients with Crohn's disease. Gastroenterology 2013;144:S86. 